\title{
Conclusions and Reflections
}

Abstract In this concluding section, Wallace brings together the evidence and analysis to argue that the devolved legislatures are engaged in a complex process of policy diffusion and policy learning, among themselves and as part of the global movements on wellbeing and sustainable development. She argues that wellbeing frameworks are an example of the non-rational, non-linear nature of policy development. That these initiatives developed during the first phase of devolution for Scotland, Wales and Northern Ireland is not a coincidence, they are best understood as a mechanism for codifying what the governments stand for (their values) and their role in relation to the UK state. With economic wellbeing largely remaining within the competency of the UK government, the devolved legislatures have chosen to implement frameworks that highlight areas that they have direct powers over. In communicating their values to the people, these new governments are also communicating their value to the people.

Keywords UK - Devolution • Policy learning - Policy diffusion • Wellbeing · Sustainable development

The research for this book raised interesting questions for me about the relationships between the three devolved jurisdictions, and the extent to which they inspire and learn from each other, as well as providing insight into their relationship with the UK state. This concluding chapter

(C) The Author(s) 2019

J. Wallace, Wellbeing and Devolution, Wellbeing in Politics and Policy, https://doi.org/10.1007/978-3-030-02230-3_7 
considers these issues in more detail and explores the extent to which the devolved governments use of wellbeing as a frame amounts to a rethinking of their role as developing legislatures.

\section{Policy Transfer, Policy Learning and Policy Diffusion}

In the early days of devolution it was argued that the new governments would create 'laboratories of democracy' with each experimenting and learning from the others (Birrell 2010). In practice, the constantly evolving nature of devolution in the UK limits the opportunity for both policy learning (taking findings from other jurisdictions and applying them to your own) and policy transfer (implement a policy that one of the other jurisdictions has tried out first) (for a sound discussion see Paul Cairney's blog, Cairney 2018).

Focused on their own internal priorities, interviewees report that there is simply limited space in the civil service timetable to keep up with the literature in other jurisdictions or make study trips to visit practice elsewhere in the UK. Given the conditions, it is perhaps surprising that there is as much policy learning as there currently is. A study of over 200 policy-makers and practitioners carried out by the Carnegie UK Trust found that seven out of 10 say that they have a working knowledge of evidence from their sector from the rest of the UK and the same proportion say evidence from their sector from the rest of the UK influences their work (Carnegie UK Trust 2018).

Analysing this in relation to wellbeing frameworks we can conclude that Wales, Scotland and Northern Ireland all engaged in policy learning to different degrees, but that only Northern Ireland adopted policy transfer-taking the policy of a national performance framework from Scotland and implementing it in their own jurisdiction. As Simon Hamilton MLA told me:

It was incredibly helpful that Scotland had taken the first steps and that it had been a success, there was a good positive example right on our doorstep. If it had been somewhere else in Europe or the far side of the world, I don't think it would have caught on this quickly.

Neither policy learning, nor policy transfer, happens organically. A key mechanism for policy learning is through inviting speakers from elsewhere to attend policy events. Experts from Wales and Scotland were 
invited to events in Northern Ireland during the development phase (2014-2016) to share their experiences with a local audience, and this did appear to have an impact on their thinking.

But the most critical factor in the policy transfer from Scotland to Northern Ireland was the study trip taken by the Carnegie Roundtable on Measuring Wellbeing in Northern Ireland to meet with Scottish stakeholders, including John Swinney MSP (then Cabinet Secretary for Finance). This was referred to by Aideen McGinley and Simon Hamilton as a 'turning point' in the process of considering a wellbeing approach. Later in the development, this was solidified by former permanent secretary Sir John Elvidge providing expert advice and support to the Northern Ireland Civil Service through a series of internal workshops. There were examples too of local authority staff sharing experience from Scotland to Northern Ireland.

There are far fewer examples of policy learning between Scotland and Wales. There was some learning around the central-local dynamic around 2010 , but this did not continue into a shared dialogue on wellbeing frameworks. This perhaps reflects the different origins of their wellbeing frameworks. It may have taken some time for actors in each of these jurisdictions to realise that they were on a shared journey.

Scotland and Wales may not have directly influenced each other at the outset but they were heavily influenced by international developments. In Scotland, the inspiration was Virginia Performs (one of a set of statelevel performance initiatives that developed in the early 2000s), solidified by study trips from the senior staff in Virginia. In Wales, the inspiration was the Millennium Development Goals and specifically the global consultation on The World We Want.

Nevertheless, we can see clearly where improvements have been made based on learning between the three jurisdictions:

- Negative lesson-drawing: the need to build a movement of support behind wellbeing as the purpose of government (Scotland to Northern Ireland).

- Positive lesson-drawing: local government alignment (Scotland to Wales), linking directly to the UN Sustainable Development Goals (Wales to Scotland); utilising audit capacity (Wales and Scotland to Northern Ireland). 
But the above analysis perhaps takes too linear a model of policy learning and transfer. It is more likely that outside of a very small number of close relationships between senior civil servants where direct policy learning took place, we are generally observing a process of policy diffusion. Policy diffusion originated in the United States as a term that summarised how different American states would emulate each other. This has similarities with the UK as the States in America, like the devolved jurisdictions in the UK, have a shared culture, history and language. In this context, ideas disperse into the policy atmosphere, creating a climate of opinion. Ideas can therefore be contagious without it being clear exactly how such information was gathered or where the inspiration originally came from (Stone 2017). For example, in reflecting on the decision to name the consultation on wellbeing in Wales a 'national conversation' Peter Davies (former Sustainable Futures Commissioner for Wales) commented in his interview: 'I do not remember the origins of the positioning as a 'national conversation' but was requested by the Minister to lead it.' National conversations were by this point in time, becoming rather common in Scotland where they had been held on constitutional change (2007-2009) and later on Fairer Scotland (2015) and Healthier Scotland (2015).

As Birrell notes, while mechanisms exist to connect civil servants, there is no formal mechanism for policy learning between politicians across the UK (2010). The closest such body is the British-Irish Council, established under the Good Friday Agreement which provides a forum for the UK and Irish Governments, the devolved governments of Northern Ireland, Scotland and Wales and the governments of the Isle of Man, Jersey and Guernsey to come together. The role is to exchange information and cooperate on matters of mutual interest. The work programme to date has focused on clear areas of policy development, such as early years, housing and creative industries, rather than cross-sectoral issues such as public sector reform. While the First Ministers of Scotland and Wales, and the An Taoiseach of the Irish Government tend to attend, the UK is most often represented by the Secretary of State for Northern Ireland and Ministers with relevant portfolios.

In their review of evidence exchange, the Alliance for Useful Evidence made several recommendations to improve sharing of learning across the UK including increased interchange of staff between the four governments and more networks of officials from the four governments that work on similar issues (Paun et al. 2016). The experience of the 
wellbeing frameworks would take this further and bring in other actors, including the independent funding sector and not-for-profit organisations who occasionally instigated as well as supported the initiatives.

\section{From Advocacy and Policy Change to Social Change?}

In assessing the impact of wellbeing frameworks in devolved governments, it quickly becomes apparent that the evidence of impact is problematic. There are few academic studies of the impact. In the case of Northern Ireland this is understandable, it is a very small jurisdiction and has only relatively recently experimented with wellbeing. It is less clear why there are so few studies of the impact in Scotland and Wales.

Those analyses that do exist come from the grey literature and these tend to take interviewees' comments at face value, so an approach is 'transformative' or 'revolutionary' because it has been called such by a stakeholder, not because of clear evidence of impact. And clear evidence of impact is hard to find. There are two potential reasons for this: the frameworks themselves are not having the desired impact; or there are impacts, but they cannot easily be traced to the wellbeing framework.

The idea of a clear linear connection between wellbeing frameworks and decisions is to misunderstand the policy process itself. Rationalist policy analysis argues that all potential options should be considered and weighed up before coming to a decision. And yet we know this is not the case. Practicalities mean governments have limited time for such options appraisals. The party-political process of producing a manifesto for government is hardly scientific or rational. Wellbeing frameworks themselves are an example of the non-rational nature of policy development. Key stakeholders have admitted that they were not entirely sure what they were doing when they started the initiatives. They had analysed the problem and considered the issues, but there was no clarity over what to do next.

Power analysis also asks us to consider what options never make it to the table. The issue of the extent to which each jurisdiction is taking a wellbeing approach tended to be solidified in the annual budget scrutiny undertaken by parliamentary committees in Scotland and Wales. But this formal decision-making comes far down the line of policy formulation. It is far harder to analyse the extent to which potential solutions are framed by contemporary narratives. The process of the controversial M4 relief road is instructive here, the decision is between two routes, the blue 
route and the black route. As Jenny Rathbone AM notes, the discussion was never open to other options: 'we don't know what the alternative ways are of spending £1.5bn to resolve the identified problem of congestion on the M4 (National Assembly for Wales 2018, online).

Given the new analysis carried out for this book, each jurisdiction can clearly tell a story of the contribution made by wellbeing frameworks to impact at the various levels (advocacy, policy and social change). For some, such as the Scottish Justice Strategy, while we cannot rule out other factors, the evidence is strong enough to attribute at least some of the social change to the framework itself. More commonly, the impact is indirect. In these cases, there is an argument to be made around the potential contribution of the framework to policy decisions but it has to be probed for, it is not offered clearly by those involved in the policy development.

Why might this be the case? Part of the explanation may lie in the way the framework influences decision-making. Several interviewees commented that it changed conversations, focusing them on the difference that is being made to citizens. They talk about asking questions of themselves and others when using the framework. Questions like: Why are we doing this? What difference are we actually making? What else is going on bere that is stopping the change from happening?

Identifying changes in decisions being made is problematic precisely because of the nature of the change in the decisions themselves: 'It's changed the nature of the dialogue. I can be absolutely categorical about that, at all levels of government, across government departments, at local and national level. It's the way in which conversations are framed-it's a mindset change' (Peter Davies, former Sustainable Futures Commissioner Wales, interview).

In assessing the wellbeing framework, we cannot do so in isolation of other changes, nor can we argue a clear linear cause and effect. Speaking at a conference in Northern Ireland on the impact of the NPF, Professor Carol Tannahill argued that the framework has helped to align people in the same direction, but it was not enough as 'other stars need to align' (Carnegie UK Trust 2017, p. 5). These other stars gather around the components of a wellbeing approach.

To understand the relationship between the wellbeing framework and the wellbeing approach, we need to avoid the suggestion of linearity and instead look at the roles being played. A wellbeing approach summarises a number of changes to how government thinks about its role and how it achieves outcomes for people. The wellbeing framework is how that paradigm shift is communicated to policy-makers, professionals and the 
public. The two exist together: without a drive to change the framing of governance, a wellbeing framework would be nothing more than an interesting way of presenting social indicators.

The analysis in this book identifies strengths and weaknesses in each jurisdiction. What might fruitful areas for positive lesson drawing be at this stage in development of the frameworks and approaches?

Firstly, Northern Ireland may be the last of the three to implement a wellbeing framework, but it has come closest to articulating how the approach works as a golden thread through the system. The NI Executive makes consistent reference to the different layers of activity: performance accountability, population accountability and societal wellbeing. This has solidified into an approach that allows indicators and outcomes to be developed that suit services (performance), population (departments) and society (government) but not to make the mistake of confusing one with the other. The health department can be responsible for service indictors and treatment outcomes, but the health of the people (measured through life expectancy, for example) is the responsibility of government as a whole (Northern Ireland Audit Office 2018). Similar effects can be seen in Scotland and Wales, but with less clarity over the reasons for the difference and less certainty for those involved over what is expected.

Secondly, Wales may have started out from a desire to have sustainable development as a central organising principle, but it can teach the other jurisdictions about legislating for culture change. The Well-being of Future Generations Act 2015 includes a set of ways of working for public bodies that are challenging to the dominant model of public service design and delivery. They are similar of course to the approaches in Scotland and Northern Ireland, but these do not have legislative weight behind them. Further, the ability to audit and formally review progress is a powerful tool for change, alongside the softer approaches used by the Commissioner to support public bodies and to act as an advocate for future generations.

Finally, Scotland shows that there are real social impacts that can be traced to the wellbeing framework. There are people in Scotland today whose lives are better because of changes in how the justice system thinks about its contribution to society. The culture change required to achieve these improvements was unlocked and supported by the framework. And culture change itself was recognised as an essential component of the wellbeing approach and supported and invested in across the civil service. 


\section{Understanding Complexity in Wellbeing}

There is an underlying tension in the three examples of wellbeing frameworks: whose responsibility is it to improve wellbeing? The conversations that have been taking place in Scotland, Wales and Northern Ireland all start from a position that it is the primary role of governments. The clarity of their statements is to be celebrated.

Underneath this overall vision however, there are tensions. The introduction to this book set out the concept of a wellbeing approach as a mechanism for whole-of-government thinking, but the whole has its bounds at the limits of government. And yet we know that much of what makes life worth living is about our relationships: our families, our friends, our community. This space, the private and the civic, is by its very nature, non-governmental.

At a societal level, we cannot hold one part of the system responsible for outcomes. For example, given what is known of the social determinants of health, it is not possible to hold the health service solely responsible for health outcomes, ignoring the known contribution of education, housing, employment and welfare to health outcomes. If we take a well-known example, life expectancy is increasing due to large reductions in smoking levels. The biggest single causal factor is the ban on smoking in public places. But other factors also contribute, smoking cessation groups, for example, and the widespread availability of e-cigarettes and vaping as a socially acceptable alternative.

We can take this a step further and see that while we can reward the governments for the improvements in health caused by the reduction in smoking, doing so removes the contribution of the market (in producing a viable alternative to smoking) and individual citizens who themselves took the step of stopping smoking. If governments were to be truly transformative in their approach, they would also recognise the contribution of the people. As discussed in Chapter 5, the governments of Scotland, Wales and Northern Ireland have stopped short of this transformation.

If the framework is owned by the government, then it is responsible for it in its entirety. But if the language shifts to the 'we' being the people, then accountability is also shared, with government seen as only contributing to social progress rather than owning it. Such a shift would require a new politics, not just a new approach to governance. 


\section{A New Politics for Wellbeing}

For Scotland, Wales and Northern Ireland, the development of a wellbeing framework was a natural step forward in their devolution journey. In each jurisdiction, stakeholders talked of the promise of devolution and the lack of progress made on improving outcomes in the intervening years. The devolved jurisdictions all have relatively small populations and systems that encourage (or in Northern Ireland's case require) coalition government. The size of the population, the relative size of the machinery of politics and government and the close relationships that exist between all parts of the system may have contributed to a shared diagnosis of the core problem-years of additional funding, and greater democratic accountability, had led to much activity but painfully little by way of change to people's actual wellbeing.

Having identified the problem, the key stakeholders in each jurisdiction set about finding solutions. Here individual politicians played significant roles in moving forward the wellbeing framework. In the debates in the Scottish Parliament, initially on the Humankind Index and then on the National Performance Framework, parliamentarians noted the cross-party support for moving beyond GDP: 'The fact that the debate is being held today is significant, as is the strength of cross-party support, along with support beyond the chamber, that it has secured' (Claudia Beamish, MSP, Labour (Scottish Parliament 2012, online)). The 2018 membership of the Cabinet Secretary's Roundtable on the National Performance Framework includes active membership from the Scottish Green Party, the Scottish National Party, Scottish Labour and the Scottish Conservatives. The developments in Northern Ireland were consciously designed to be cross-party, indeed as Simon Hamilton MLA told me: 'If you didn't have cross-party agreement you wouldn't have got anywhere'. The experience in Wales was slightly bumpier, with disagreements relating to the balance between economic and environmental issues and the balance between sustainable development and public sector reform. The core aim of legislating for a sustainable development principle did not however appear controversial.

What could inspire such unanimity of purpose?

For much of this analysis, I have referred to the devolved governments as though they have little or no relationship to their 'parent' Parliament in Westminster. In doing so, I followed the evidence from written sources and interviewees, parliamentary debates and reports which refer to 
Westminster only rarely, and only to note that the devolved approach is different (and by strong implication, better). The desire to do things differently appears in many policy conversations in the devolved jurisdictions.

The four legislatures of the UK (UK, Scotland, Wales and Northern Ireland) are engaged in a complex system of their own. Scotland and Wales seek to mark out their differences from Whitehall, Northern Ireland aligns with the UK politically but seeks to assert itself as a mature government by emulating Scotland and Wales.

The economic and welfare policy responsibilities that remain within the competency of Westminster have a substantial effect on wellbeing. There is an argument therefore that rather than rebalancing the dominance of the economic model on government decision-making, what the devolved governments are actually saying is that they wish to accentuate the areas of wellbeing over which they have control. The domains of social and environmental wellbeing are broadly within devolved competency. Do devolved jurisdictions highlight these due to sustainable development arguments, or are they highlighting areas where they have direct powers under the constitutional settlements? If this is the case, it is not a damning criticism. Devolved administrations can make little difference to people's lives where they do not have real powers.

As the devolution settlements evolve further, and devolution of powers on the economy seems set to grow and mature, this logic may alter. At the time of writing (June 2018), the extent to which the UK Government will retain the powers it is taking back from the European Union or will allow the devolved legislatures to take them directly is unclear. But the effect of Brexit on intra-UK relationships is beginning to crystallise suggesting a tense time ahead for the relationships between the different tiers of governance in the UK.

For now, there remains a riddle at the heart of a devolved administration's approach to wellbeing: why would a government seek accountability for outcomes they cannot directly affect? Part of the answer to this question may lie in the concept of a framework for government itself. In the maturing of their approach, the devolved legislatures are seeking to articulate that they stand for the common good and hold within themselves the values of the society that they govern. It is no surprise that these smaller and newer legislatures have sought to codify what they stand for. It is no surprise that they have done so in ways that bind each other to a shared set of outcomes. In communicating their values to the people, they are also communicating their value to the people. 


\section{REFERENCES}

Birrell, D. (2010). Devolution and Approaches to Social Policy. Devolution in Practice 2010 (pp. 125-140). London: IPPR.

Cairney, P. (2018). Policy Concepts in 1000 Words-Policy Transfer and Learning [Online]. https://paulcairney.wordpress.com/2013/11/11/ policy-concepts-in-1000-words-policy-transfer-and-learning/.

Carnegie UK Trust. (2017). Towards a Wellbeing Framework: From Outcomes to Actions (Conference Report). Dunfermline: Carnegie UK Trust.

Carnegie UK Trust. (2018). Evidence Exchange [Online]. Available at: https:// www.carnegieuktrust.org.uk/carnegieuktrust/wp-content/uploads / sites/64/2018/03/Evidence-Exchange-infographic.pdf. Accessed 2018.

National Assembly for Wales. (2018). Equality, Local Government and Communities Committee [Online]. Available at: http://record.assembly. wales/Committee/4589\#C66284. Accessed 2018.

Northern Ireland Audit Office. (2018). Performance Management for Outcomes. Belfast: Northern Ireland Audit Office.

Paun, A., Rutter, J., \& Nicholl, A. (2016). Devolution as a Policy Laboratory: Evidence Sharing and Learning Between the UK's Four Governments. London: Alliance for Useful Evidence.

Scottish Parliament. (2012). Official Report: Meeting of the Parliament 05 September 2012 [Online]. Available at: http://www.parliament.scot/parliamentarybusiness $/$ report.aspx $? \mathrm{r}=7524 \& \mathrm{i}=68637 \& \mathrm{c}=0 \& \mathrm{~s}=$ humankind. Accessed 2018.

Stone, D. (2017). Understanding the Transfer of Policy Failure: Bricolage, Experimentalism and Translation. Policy \& Politics, 45(1), 55-70. 
Open Access This chapter is licensed under the terms of the Creative Commons Attribution 4.0 International License (http://creativecommons.org/licenses/ by $/ 4.0 /$ ), which permits use, sharing, adaptation, distribution and reproduction in any medium or format, as long as you give appropriate credit to the original author(s) and the source, provide a link to the Creative Commons license and indicate if changes were made.

The images or other third party material in this chapter are included in the chapter's Creative Commons license, unless indicated otherwise in a credit line to the material. If material is not included in the chapter's Creative Commons license and your intended use is not permitted by statutory regulation or exceeds the permitted use, you will need to obtain permission directly from the copyright holder.

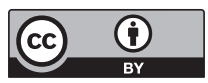

